\title{
Flux thermique et coefficients de transfert global et partiel d'un échangeur à ailettes spiralées en graphite
}

\author{
Zina Meddeb ${ }^{1, a}$, Mohamed Razak Jeday ${ }^{1}$ et Souad Harmand ${ }^{2}$ \\ 1 Laboratoire d'Énergétique et d'Ingénierie, École Nationale d'Ingénieurs de Gabès, Route de Medenine, 6029 Gabès, Tunisie \\ 2 Laboratoire de Mécanique et d'Énergétique, Université de Valenciennes et de Hainaut Cambrésis, Le Mont Houy, \\ 59313 Valenciennes Cedex 09, France
}

Reçu le 28 mars 2003, accepté le 5 mars 2004

\begin{abstract}
Résumé - La technique d'évaporation présentée dans ce travail consiste à faire ruisseler un liquide à évaporer à la surface d'ailettes enroulées en spirale autour d'un tube vertical, chauffé intérieurement par un fluide caloporteur. Le tube et les ailettes sont usinés dans un monobloc massif en graphite imprégné. Compte tenu de la grande complexité de la géométrie de cet échangeur et de l'écoulement sur ses ailettes, la détermination du coefficient de transfert thermique par convection externe est difficile à obtenir par un calcul direct. Il est déterminé ici par voie indirecte en suivant la méthode de Wilson basée sur deux étapes : une expérimentale et l'autre théorique. On détermine dans cet article la quantité de chaleur échangée et la conductance globale au transfert thermique de cet échangeur/évaporateur lors de l'échauffement. On détermine également les différentes résistances partielles ainsi que le coefficient de convection entre la paroi externe de l'ailette et le film de la solution à chauffer. La démarche proposée dans cet article a permis de valider la méthode indirecte.
\end{abstract}

Mots clés : Thermique / échangeur / ailette / coefficient d'échange global / convection

\begin{abstract}
Heat flux and global and partial heat transfer coefficient in an exchanger with helicoidal graphite fins. A new evaporation technique consists of letting a stream of liquid flow along an helicoidally fins. The fins that are rolled in spirals around a heated vertical tube through with a hot fluid is circulated. The tube and fins are manufactured one bloc of impregnated graphite. Because of the great complexity of the geometry of this exchanger and the flow on its fins, the determination of the thermal transfer coefficient by external convection is difficult to obtain by a direct calculation. It is indirectly determined here by Wilson's method which is based on two stages: one is experimental and the other is theoretical. In this article, we determine the quantity of exchanged heat and the coefficient global of the thermal transfer of this exchanger/evaporator during the heating, also, we determine the different partial resistances during the heating, as well as the coefficient of convection between the external wall of the fin and the solution to heat. The procedure, proposed in this work, has allowed to validate the equation proposed by Wilson.
\end{abstract}

Key words: Thermal / exchanger / fin / global exchange coefficient / convection

\section{Introduction}

La fabrication de l'acide phosphorique passe par un stade de concentration de $28 \%$ à $54 \%$ en $\mathrm{P}_{2} \mathrm{O}_{5}$. Pour concentrer l'acide on élimine une quantité d'eau de dilution par vaporisation sous vide. Les procédés industriels de concentration sont similaires et ils comprennent essentiellement un échangeur thermique, un bouilleur,

a Auteur correspondant : Zina.Meddeb@issatgb.rnu.tn un condenseur barométrique et une pompe qui assure la circulation de l'acide du bas vers le haut. Ces procédés présentent beaucoup de problèmes tels que le bouchage des tubes, vu que l'acide phosphorique est un produit très encrassant, et des pertes énergétiques vu l'existence de gradients importants de température et de pression entre l'échangeur et le bouilleur [1].

Une solution consiste à remplacer l'échangeur et le bouilleur par un seul élément, dans lequel l'acide circule du haut vers le bas par gravité. Il s'agit d'un 


\section{Nomenclature}

\begin{tabular}{|c|c|c|}
\hline$A$ & surface d'échange thermique & $\mathrm{m}^{2}$ \\
\hline$b$ & constante & \\
\hline$C_{p f}$ & chaleur massique de la solution & $\mathrm{J} . \mathrm{kg}^{-1} \cdot \mathrm{K}^{-1}$ \\
\hline$D e$ & diamètre extérieur du tube & $\mathrm{m}$ \\
\hline$D b$ & diamètre de base du tube & $\mathrm{m}$ \\
\hline$D i$ & diamètre intérieur du tube & $\mathrm{m}$ \\
\hline Epe & épaisseur à l'extrémité & $\mathrm{m}$ \\
\hline Epo & épaisseur à la base & $\mathrm{m}$ \\
\hline$F$ & facteur correctif & \\
\hline$G c$ & débit massique de la vapeur & $\mathrm{kg} \cdot \mathrm{s}^{-1}$ \\
\hline$G f$ & débit massique de la solution & $\mathrm{kg} \cdot \mathrm{s}^{-1}$ \\
\hline Ho & hauteur du tube & $\mathrm{m}$ \\
\hline$h g f$ & coefficient de convection & $\mathrm{W} \cdot \mathrm{m}^{-2} \cdot \mathrm{K}^{-1}$ \\
\hline $2 \pi h$ & pas de l'ailette & $\mathrm{m}$ \\
\hline$L a$ & largeur de l'ailette & $\mathrm{m}$ \\
\hline$M f$ & débit volumique de la solution & $1 . h^{-1}$ \\
\hline$N$ & nombre d'ailettes par tube & \\
\hline$k$ & constante & \\
\hline$R j f$ & résistance thermique globale & $\mathrm{K} . \mathrm{W}^{-1}$ \\
\hline$R j i$ & résistance thermique interne & $\mathrm{K} . \mathrm{W}^{-1}$ \\
\hline Rig & résistance thermique du graphite & K. $\mathrm{W}^{-1}$ \\
\hline$R g f$ & résistance thermique externe & $\mathrm{K} . \mathrm{W}^{-1}$ \\
\hline$T_{\mathrm{c}}$ & température de la vapeur caloporteur & $\mathrm{K}$ \\
\hline$T_{\mathrm{f}}$ & température de la solution & $\mathrm{K}$ \\
\hline$U g$ & conductance globale d'échange & $\mathrm{W} \cdot \mathrm{m}^{-2} \cdot \mathrm{K}^{-1}$ \\
\hline \multicolumn{3}{|c|}{ Symboles grecs } \\
\hline$\alpha 1$ & inclinaison de la face supérieure & $\left({ }^{\circ}\right)$ \\
\hline$\alpha 2$ & inclinaison de la face inférieure & $\left({ }^{\circ}\right)$ \\
\hline$\beta$ & inclinaison de l'ailette & $\left({ }^{\circ}\right)$ \\
\hline$\lambda$ & conductivité thermique du graphite & $\mathrm{W} \cdot \mathrm{m}^{-1} \cdot \mathrm{K}^{-1}$ \\
\hline$\Delta T m l$ & température moyenne logarithmique & \\
\hline$\Phi$ & flux thermique & $\mathrm{W}$ \\
\hline
\end{tabular}

nouvel échangeur/évaporateur (proposé en collaboration avec l'équipementier Carbone Lorraine, France) où s'effectuent l'échauffement et l'évaporation à la fois. L'évaporation commence si la température de la solution en écoulement atteint la température de changement de phase [2]. La présente étude concerne l'utilisation de ce type d'échangeur pour le chauffage de l'acide phosphorique, néanmoins, ce procédé peut être utilisé pour chauffer ou évaporer d'autres solutions.

La nouvelle technique étudiée [3] qui n'est pas encore mise en œuvre, consiste à faire ruisseler le liquide à chauffer ou à évaporer à la surface d'ailettes enroulées en spirales autour d'un tube vertical chauffé intérieurement par un fluide caloporteur. Le tube et les ailettes sont usinés à partir d'un monobloc massif en graphite imprégné.

La figure 1 donne un schéma de principe de fonctionnement. Un tel appareil est caractérisé par de nombreux paramètres géométriques dont les principaux sont donnés sur la figure 2 .

Afin d'évaluer les caractéristiques thermiques de ce nouveau type d'échangeur, dans ce travail nous allons d'une part appréhender l'influence de l'aspect hydrodynamique du film sur le flux de chaleur échangé et sur le coefficient global d'échange thermique et d'autre part, déterminer le coefficient partiel d'échange thermique convectif ailette-fluide à chauffer.

Compte tenu de la grande complexité de la géométrie du nouvel échangeur et de l'écoulement sur ses ailettes, la détermination du coefficient de transfert thermique par convection externe est difficile à obtenir par un calcul direct. Il est déterminé dans ce travail par voie indirecte en suivant une méthode en deux parties : une expérimentale et l'autre théorique. 


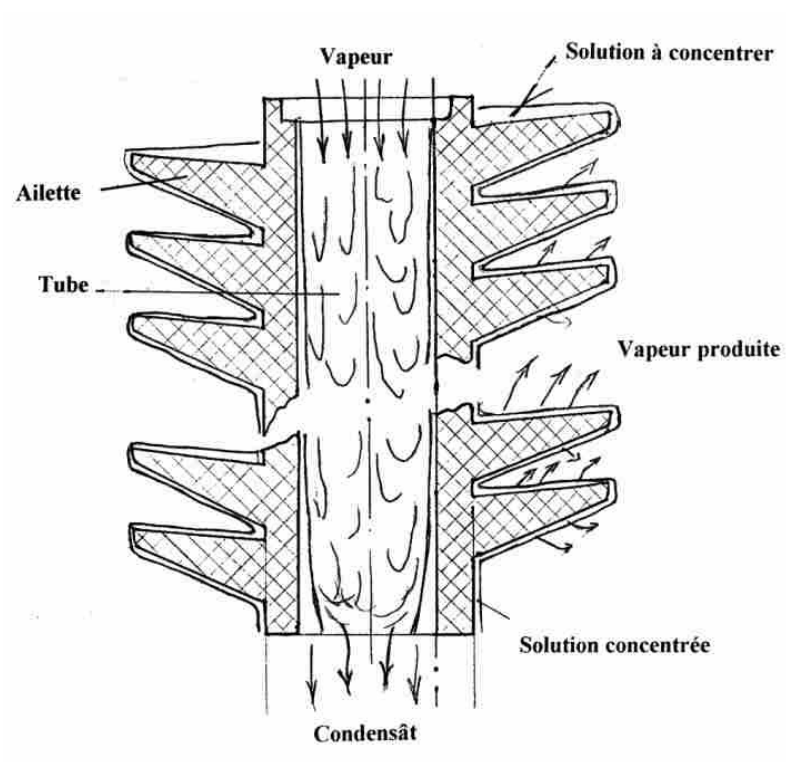

Fig. 1. Schéma de principe de fonctionnement du nouvel échangeur/évaporateur.

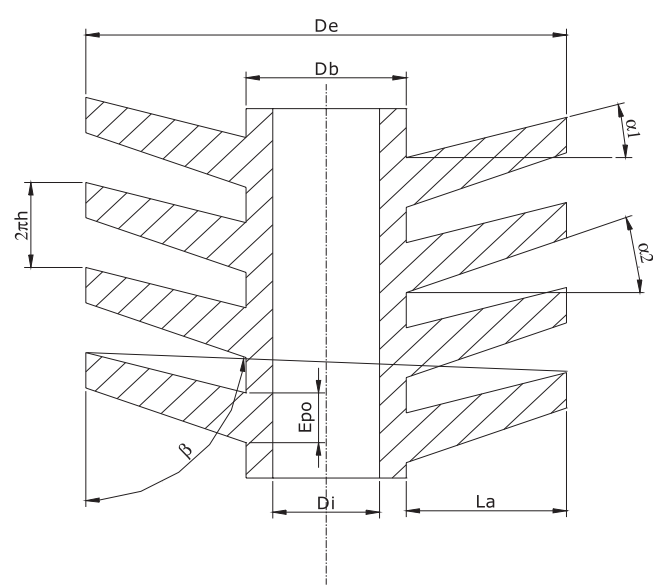

Fig. 2. Schéma de l'échangeur/évaporateur à ailettes spiralées.

\section{Conditions et dispositifs expérimentaux}

\subsection{Conditions expérimentales}

Les essais sont effectués dans les conditions suivantes :

- L'étude est faite en régime permanent.

- La circulation de la solution et du fluide caloporteur se font à co-courant du haut vers le bas.

- Le chauffage de la solution est effectué sans changement de phase sous la pression atmosphérique.

- Les solutions à chauffer sont l'eau de la ville de Gabès et l'acide phosphorique industriel à deux concentrations massiques différentes : $28 \%$ et $54 \%$ en $\mathrm{P}_{2} \mathrm{O}_{5}$.

- Les fluides caloporteurs utilisés sont :

- l'eau chaude pour la solution d'acide phosphorique à $54 \%$ en $\mathrm{P}_{2} \mathrm{O}_{5}$;

- la vapeur d'eau à l'état de saturation pour l'eau de la ville de Gabès et pour la solution d'acide phosphorique à $28 \%$ en $\mathrm{P}_{2} \mathrm{O}_{5}$.

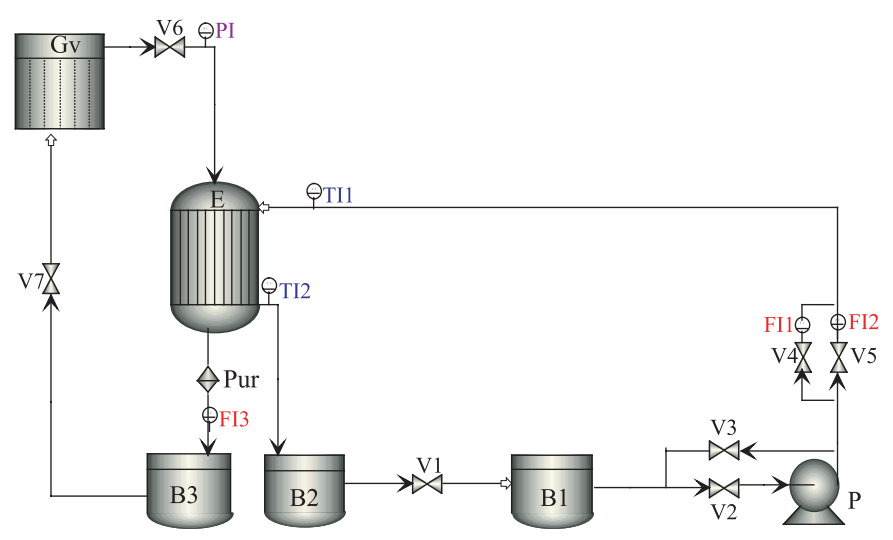

Fig. 3. Schéma de principe de l'installation.

\subsection{Dispositifs expérimentaux}

Pour réaliser les essais permettant d'atteindre les objectifs visés dans ce travail, nous avons réalisé une installation munie de l'instrumentation nécessaire (Fig. 3). Elle comporte :

- l'échangeur/évaporateur (E);

- un générateur de vapeur $(\mathrm{Gv})$;

- des bacs d'alimentation ou de récupération (B1), (B2) et (B3) ;

- une pompe d'alimentation de la solution à concentrer $(\mathrm{P})$;

- un purgeur (Pur) et des vannes de réglage (Vi, $i=1$ à 7$)$;

- des indicateurs des débits de la solution à chauffer à l'entrée de l'échangeur (FI1) et (FI2). Pour chaque gamme de débits nous avons utilisé le débitmètre adéquat pour une meilleur précision;

- un indicateur de débit de condensat de fluide de chauffe (FI3) ;

- deux indicateurs de températures de la solution à chauffer une à l'entrée et l'autre est à la sortie de l'échangeur (TI1) et (TI2);

- un indicateur de la pression de la vapeur de chauffe à l'entrée de l'échangeur (PI).

Les paramètres géométriques de la maquette expérimentale sont regroupés dans le tableau 1.

L'échangeur/évaporateur est équipé d'une calandre en verre permettant de visualiser les différents régimes hydrodynamiques et de retenir les éventuels projections de la solution. La mesure des températures aux principaux points de l'installation est faite en utilisant des thermocouples de type Chromel/Alumel. Les débits des solutions sont contrôlés par deux rotamètres à flotteurs classiques.

Concernant cette démarche expérimentale il faut remarquer que :

1. Nous avons utilisé l'eau chaude comme fluide de chauffage pour éviter les problèmes d'étanchéité. Mais pour la détermination du coefficient de convection thermique nous avons utilisé comme fluide de chauffage la vapeur d'eau saturée, parce que la méthode utilisée exige que le flux thermique provienne de la condensation de la vapeur de chauffe. 
Tableau 1. Caractéristiques géométriques de la maquette expérimentale de l'échangeur/évaporateur à ailettes spiralées.

\begin{tabular}{|c|c|c|c|c|c|c|c|c|c|c|c|}
\hline $\begin{array}{c}D e \\
(\mathrm{~mm})\end{array}$ & $\begin{array}{r}D b \\
(\mathrm{~mm})\end{array}$ & $\begin{array}{r}D i \\
(\mathrm{~mm})\end{array}$ & $\begin{array}{r}H o \\
(\mathrm{~mm})\end{array}$ & $\begin{array}{r}2 \pi h \\
(\mathrm{~mm})\end{array}$ & $\begin{array}{r}L a \\
(\mathrm{~mm})\end{array}$ & $\begin{array}{r}\text { Epe } \\
(\mathrm{mm})\end{array}$ & $\begin{array}{r}\text { Epo } \\
(\mathrm{mm})\end{array}$ & $N$ & $\begin{array}{l}\alpha 1 \\
\left({ }^{\circ}\right)\end{array}$ & $\begin{array}{l}\alpha 2 \\
\left({ }^{\circ}\right)\end{array}$ & $\begin{array}{r}\beta \\
\left({ }^{\circ}\right)\end{array}$ \\
\hline 140 & 60 & 30 & 500 & 35 & 40 & 8 & 20 & 14 & 10 & 25 & 82,9 \\
\hline
\end{tabular}

Tableau 2. Domaines de présence des différents régimes d'écoulement pour l'eau à $299 \mathrm{~K}$, pour l'acide phosphorique à $28 \%$ en $\mathrm{P}_{2} \mathrm{O}_{5}$ à $302 \mathrm{~K}$ et pour l'acide phosphorique à $54 \%$ en $\mathrm{P}_{2} \mathrm{O}_{5}$ à $343 \mathrm{~K}$.

\begin{tabular}{|c|c|c|c|}
\hline \multirow[b]{2}{*}{$\begin{array}{l}\text { Régime } \\
\text { hydrodynamique }\end{array}$} & \multicolumn{3}{|c|}{ Débit de la solution à chauffer $\quad M f\left(1 . h^{-1}\right)$} \\
\hline & $\begin{array}{l}\text { Eau } \\
\text { de la ville } \\
\text { de Gabès } \\
\end{array}$ & $\begin{array}{l}\text { Acide } \\
\text { phosphorique } \\
\text { à } 28 \% \text { en } \mathrm{P}_{2} \mathrm{O}_{5}\end{array}$ & $\begin{array}{l}\text { Acide } \\
\text { phosphorique } \\
\text { à } 54 \% \text { en } \mathrm{P}_{2} \mathrm{O}_{5}\end{array}$ \\
\hline $\begin{array}{l}\text { Seules les faces supérieures } \\
\text { sont mouillées (Régime } \boldsymbol{n}^{\circ} \mathbf{1} \text { ) }\end{array}$ & $M f<60$ & $M f<65$ & $M f<75$ \\
\hline $\begin{array}{l}\text { Débordement avec des zones } \\
\text { sèches (Régime } \boldsymbol{n}^{\circ} 2 \text { ) }\end{array}$ & $60<M f<400$ & $65<M f<400$ & $75<M f<540$ \\
\hline $\begin{array}{l}\text { Toutes les ailettes sont mouillées } \\
\text { (Régime } \boldsymbol{n}^{\circ} \text { 3) }\end{array}$ & $400<M f<550$ & $400<M f<600$ & $540<M f<750$ \\
\hline $\begin{array}{l}\text { Excès de débordement } \\
\text { (Régime } \boldsymbol{n}^{\circ} \text { 4) }\end{array}$ & $M f>550$ & Mf $>600$ & Mf $>750$ \\
\hline
\end{tabular}

2. Nous avons mesuré les débits massiques des fluides soit à l'entrée soit à la sortie vu qu'il y a conservation de la matière (régime permanent).

3. Nous avons mesuré seulement les températures à l'entrée et à la sortie de l'échangeur que ce soit pour le fluide de chauffage ou pour le fluide à chauffer.

4. Si le fluide de chauffage est de la vapeur, nous avons mesuré uniquement sa pression pour en déduire sa température (étant donné qu'il s'agit d'une vapeur saturée).

5. La solution à chauffer est à l'état liquide à l'entrée et à la sortie.

6. Si le fluide de chauffage est de l'eau chaude, alors il est à l'état liquide dès l'entrée jusqu'à la sortie de l'échangeur.

7. Si le fluide de chauffage est de la vapeur saturée, alors il est sous forme de vapeur à l'entrée, sous forme d'un mélange vapeur-liquide à l'intérieur et sous forme liquide à la sortie de l'échangeur, (puisqu'il y a condensation totale de la vapeur de chauffage parce que nous avons placé un purgeur à la sortie de l'échangeur pour qu'on puisse mesurer son débit).

\section{Description des régimes d'écoulement}

Pour pouvoir identifier les différents régimes hydrodynamiques, on a fait varier graduellement le débit de la solution à chauffer $(M f)$ à l'entrée de l'échangeur tout en observant la nature hydrodynamique du film [4]. Ainsi on a pu identifier en fonction de la gamme des débits étudiés, quatre régimes d'écoulement. Les débits correspondants en fonction de la nature de la solution à chauffer

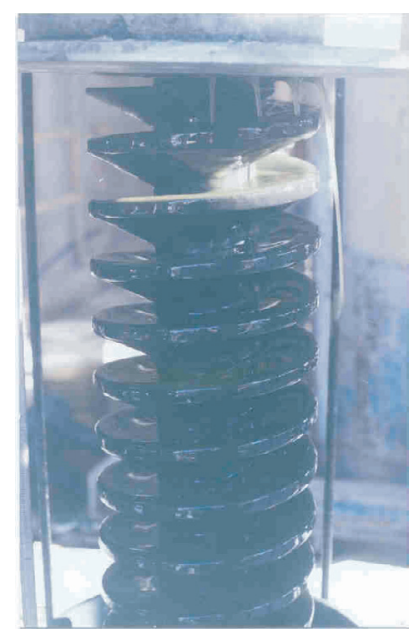

Fig. 4. Photographie du régime $\mathrm{n}^{\circ} 2$ obtenu pour un débit $M f=1001 \cdot h^{-1}$.

sont rassemblés dans le tableau 2. Les régimes hydrodynamiques identifiés sont :

- un régime où seules les faces supérieures des ailettes sont mouillées (régime 1);

- un régime avec un faible débordement (régime 2);

- un régime où les ailettes sont totalement mouillées (régime 3) ;

- un régime avec excès de débordement (régime 4).

À titre d'illustration, les figures 4 à 6 présentent respectivement des photographies du deuxième, du troisième et du quatrième régime d'écoulement, obtenus avec une solution d'acide phosphorique à $54 \%$ en $\mathrm{P}_{2} \mathrm{O}_{5}$ à la température de $343 \mathrm{~K}$. La figure 7 donne une représentation schématique de l'ensemble des régimes. 


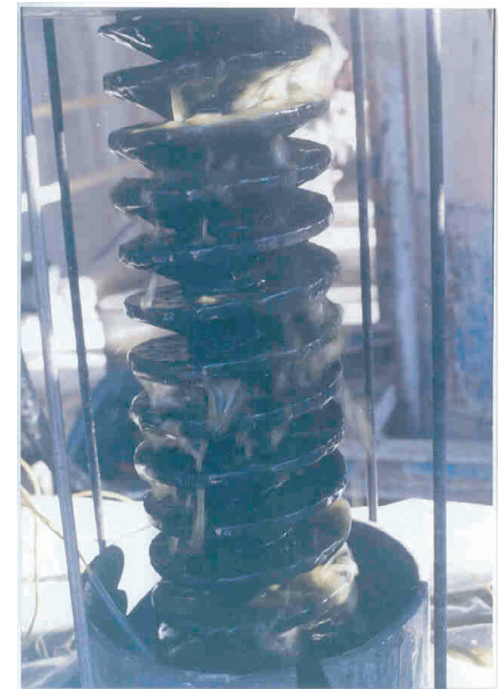

Fig. 5. Photographie du régime $\mathrm{n}^{\circ} 3$ obtenu pour un débit $M f=6001 . h^{-1}$.

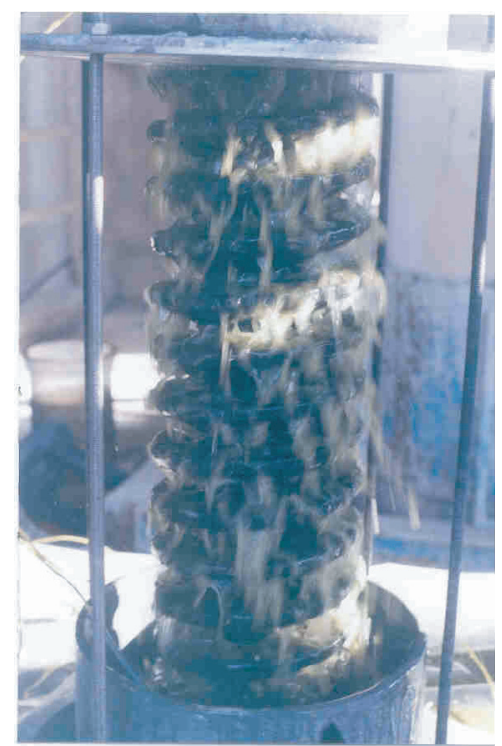

Fig. 6. Photographie du régime $\mathrm{n}^{\circ} 4$ obtenu pour un débit $M f=800 \mathrm{l} \cdot \mathrm{h}^{-1}$.

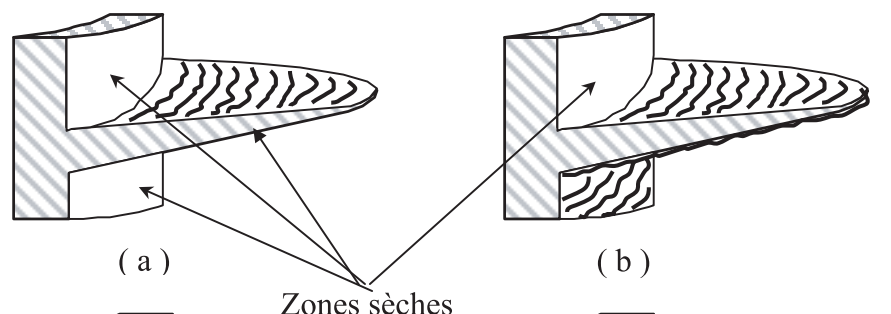

( c )

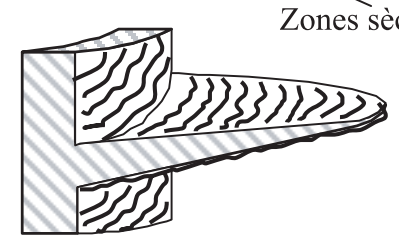

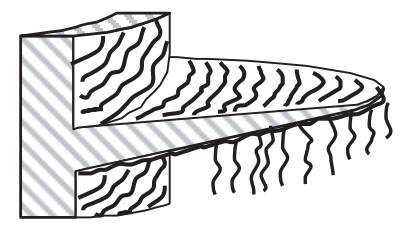

(d)
Fig. 7. Les quatre régimes d'écoulement.

\section{4 Étude thermique}

\subsection{Méthode de détermination du flux de chaleur et du coefficient global d'échange thermique}

Dans ce qui suit, nous allons présenter les relations utilisées pour calculer le flux thermique échangé, ainsi que la conductance globale d'échange thermique.

Le flux de chaleur échangé $(\Phi)$ entre le fluide de chauffage et la solution est obtenu à partir de la relation :

$$
\Phi=G_{f} \int_{T_{\mathrm{fe}}}^{T_{\mathrm{fs}}} C_{\mathrm{pf}}(T) \cdot \mathrm{d} T
$$

où $T_{\mathrm{fe}}$ est la température de la solution à l'entrée et $T_{\mathrm{fs}}$ est la température de la solution à la sortie.

Le coefficient global d'échange thermique $(U g)$ est donné par [5-7] :

$$
U g=\frac{\Phi}{\text { Aint.F. } \Delta T m l}
$$

$\Delta T m l$ étant la différence de température moyenne logarithmique, donnée par l'expression suivante :

$$
\Delta T m l=\frac{\left(T_{\mathrm{ce}}-T_{\mathrm{fe}}\right)-\left(T_{\mathrm{cs}}-T_{\mathrm{fs}}\right)}{\operatorname{Ln} \frac{T_{\mathrm{ce}}-T_{\mathrm{fe}}}{T_{\mathrm{cs}}-T_{\mathrm{fs}}}}
$$

(Aint) est la surface intérieure d'échange thermique.

Le facteur correctif $(F)$ intervenant dans la relation (2) sert à tenir compte du type d'écoulement du fluide de chauffage par rapport au fluide à chauffer [5-7].

\subsection{Méthodologie suivie pour la détermination du coefficient partiel d'échange thermique (hgf)}

La résistance globale au transfert thermique ( $R j f)$ est déterminée à partir de la conductance globale d'échange thermique en utilisant la formule suivante [7] :

$$
R j f=\frac{1}{U g \cdot \text { Aint }}
$$

La résistance globale de transfert thermique (Rjf) peut être considérée comme la somme de trois résistances partielles (Fig. 8), soit :

$$
R j f=R j i+R i g+R g f
$$

avec :

$R j i$ : Résistance interne (fluide de chauffage/paroi interne).

Rig : Résistance de conduction à travers l'ailette en graphite.

$R g f$ : Résistance externe (solution à chauffer/paroi externe).

Dans la résistance externe, on néglige l'effet de l'encrassement. 


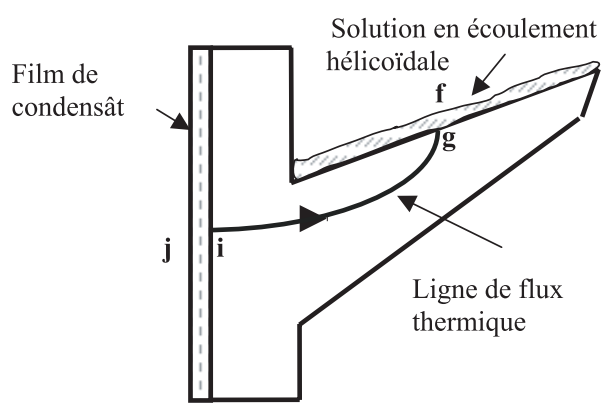

Fig. 8. Les principales résistances au transfert thermique.

Le coefficient partiel (hgf) est donné par :

$$
h g f=\frac{1}{R g f \cdot A e x t}
$$

(Aext) est la surface extérieure d'échange thermique.

En se basant sur les travaux de Wilson [8] et de Briggs $[9,10]$, nous allons supposer que la résistance interne $(R j i)$ peut être exprimée en fonction du débit du fluide de chauffage par une relation en puissance de la forme :

$$
R j i=k \cdot(G c)^{-0,8}
$$

où $G c$ est le débit du fluide caloporteur et $k$ est une constante qui dépend des caractéristiques physicochimiques du fluide de chauffage et de la géométrie de la surface d'échange.

L'équation proposée par Wilson s'applique dans le cas d'un tube à ailettes avec un transfert thermique sans changement de phase coté ailette. Le fluide de chauffage est de la vapeur d'eau et la chaleur transférée est la chaleur de condensation de cette vapeur. Cette équation ne tient compte d'aucune considération locale des températures et seuls les paramètres d'entrée et de sortie sont pris en compte.

D'autre part, l'ailette est caractérisée par son efficacité $\left(\eta_{\text {ail }}\right)$ donnée par la relation suivante $[7,11]$ :

$$
\eta_{\text {ail }}=\frac{R g f}{R i g+R g f}
$$

d'où la somme des résistances Rig et Rgf est :

$$
R i g+R g f=\frac{R g f}{\eta_{\text {ail }}}
$$

En substituant les expressions (7) et (9) dans la relation (5), on obtient :

$$
R j f=k G c^{-0,8}+\frac{R g f}{\eta_{\text {ail }}}
$$

En présentant $(R j f)$ en fonction de $(G c)^{-0,8}$ on obtient une droite de pente $k$ et d'ordonnée à l'origine $b$ égale à $\frac{R g f}{\eta_{\text {ail }}}$.

Ainsi en utilisant la relation (6), on obtient l'expression de la valeur à l'origine $b$ suivante :

$$
b=\frac{1}{h g f . A e x t . \eta_{\text {ail }}}
$$

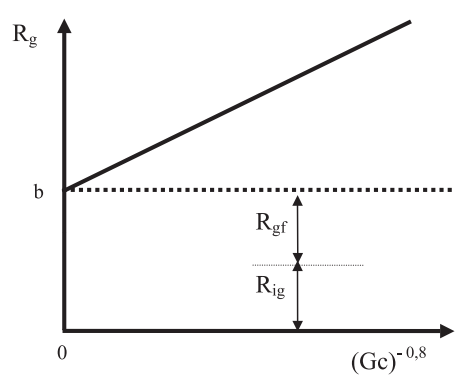

Fig. 9. Détermination indirecte de la somme des résistances de l'ailette et du film.

L'expression de l'efficacité de l'ailette peut être donnée par la formule suivante en fonction de $(h g f)[7,11,12]$ :

$$
\eta_{\text {ail }}=\frac{\operatorname{th}(X)}{X}
$$

où $(X)$ est une fonction de $(h g f)$, soit :

$$
X=\sqrt{\frac{h g f \cdot L a^{2}}{\lambda \cdot\left(\frac{E p}{2}\right)}}
$$

L'épaisseur moyenne de l'ailette est estimée par :

$$
E p=\frac{E p o+E p e}{2}
$$

La méthode indirecte pour l'évaluation des valeurs du coefficient (hgf) est donc basée sur les deux étapes suivantes.

\section{Étape $n^{\circ} 1$}

Le tracé graphique des valeurs expérimentales de la résistance globale $(R g)$ en fonction du débit du fluide de chauffage $(G c)^{-0,8}$ (Fig. 9) permet de déterminer l'ordonnée à l'origine $b$ par ailleurs égale à la somme $(R i g+R g f)$.

\section{Étape $n^{\circ} 2$}

La détermination du coefficient de convection thermique (hgf) par le calcul itératif de l'équation suivante :

$$
\frac{t h \sqrt{\frac{h g f \cdot L a^{2}}{\lambda .(E p / 2)}}}{\sqrt{\frac{h g f \cdot L a^{2}}{\lambda .(E p / 2)}}}=\frac{1}{h g f \cdot \text { Aext.b }}
$$

\section{Résultats}

La figure 10 montre la variation du flux de chaleur $(\Phi)$ en fonction du débit volumique $(M f)$ de la solution d'acide phosphorique à $54 \%$ en $\mathrm{P}_{2} \mathrm{O}_{5}$. La figure 11 donne les variations de la conductance globale d'échange thermique 


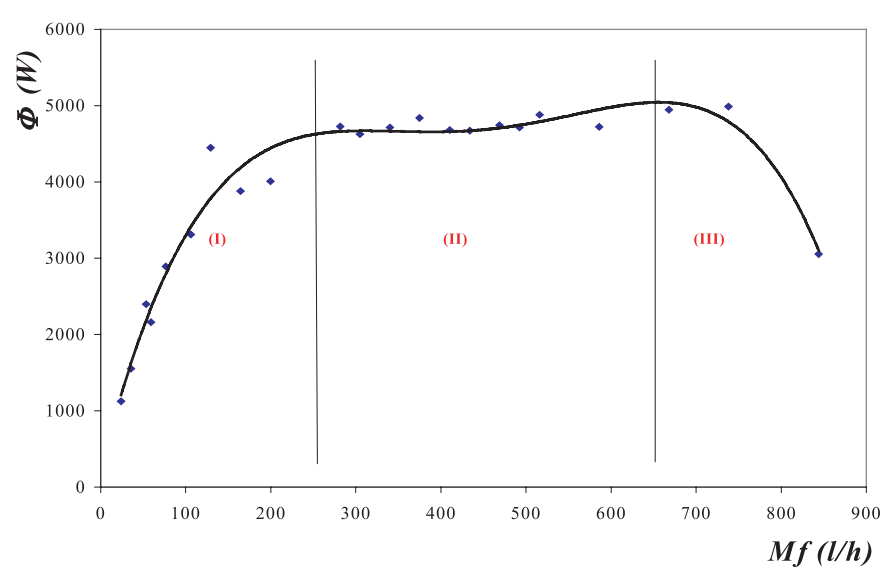

Fig. 10. Variation du flux thermique échangé en fonction du débit d'alimentation de l'acide phosphorique à $54 \%$ en $\mathrm{P}_{2} \mathrm{O}_{5}$.

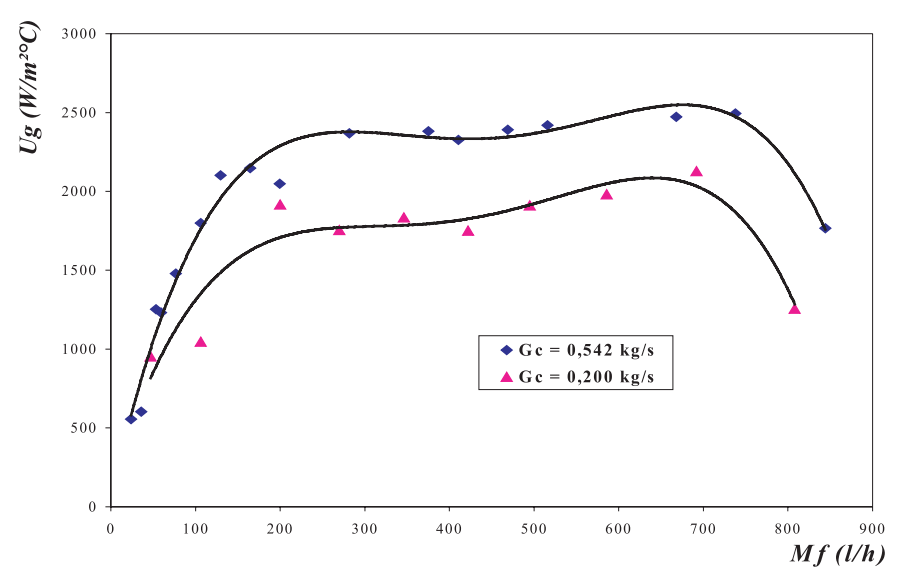

Fig. 11. Variation du coefficient global d'échange thermique en fonction du débit d'acide phosphorique à $54 \%$ en $\mathrm{P}_{2} \mathrm{O}_{5}$ pour deux débits de vapeur caloporteur.

$(U g)$ en fonction de débit volumique d'alimentation $(M f)$ pour la solution d'acide phosphorique à $54 \%$ en $\mathrm{P}_{2} \mathrm{O}_{5}$ pour deux débits de chauffage. La figure 12 présente les variations de la résistance globale $(R g)$ en fonction de $\left(G c^{-0,8}\right)$ pour différent débits de la solution d'acide phosphorique à $28 \%$. La figure 13 compare les variations du coefficient de convection ( $h g f$ ) déterminé par la méthode indirecte d'une part et déterminé expérimentalement par Soetrisnanto [13] d'autre part pour des conditions similaires.

\section{Analyse et commentaires}

\subsection{Le flux et le coefficient global d'échange thermique}

En observant le graphique de la figure 10, on note la présence des trois domaines de variations suivants.

\section{Domaine $n^{\circ}(I)$}

Dans ce domaine, la variation de $(\Phi)$ en fonction de (Mf) est fortement croissante. Cette croissance peut être

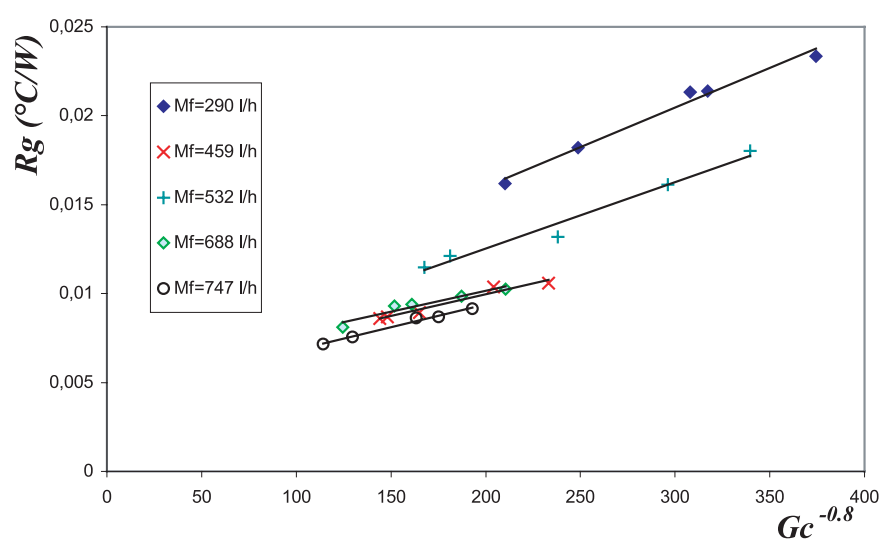

Fig. 12. Variations de la résistance globale $(R g)$ en fonction de $(G c)^{-0,8}$ pour différent débits d'acide phosphorique à $28 \%$ en $\mathrm{P}_{2} \mathrm{O}_{5}$.

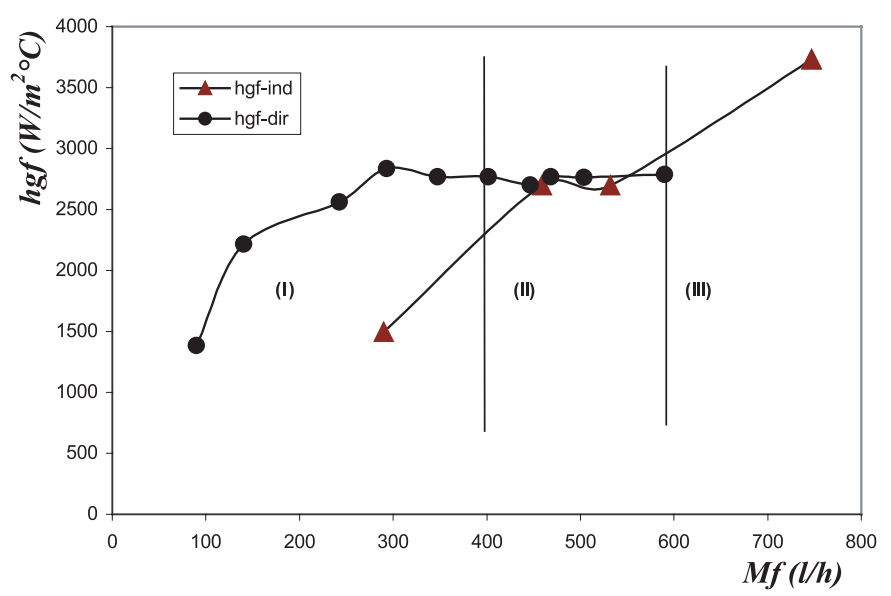

Fig. 13. Coefficient de convection $(h g f)$ par la méthode de Wilson et par mesure directe.

attribuée à l'augmentation de la surface offerte à l'échange thermique au fur et à mesure que le débit de la solution augmente (la surface mouillée est en augmentation pour les régimes hydrodynamique numéros 1 et 2 ). Ce domaine correspond ici aux valeurs de $(M f)$ inférieures à $2801 . \mathrm{h}^{-1}$.

\section{Domaine $n^{\circ}($ II)}

Il est caractérisé par une variation quasi-constante de $(\Phi)$ en fonction de $(M f)$. Ceci correspond au régime de fonctionnement où la surface externe d'échange est intégralement mouillée (régime hydrodynamique numéro 3). Ce domaine correspond à des valeurs de $(M f)$ comprises entre 280 et $6801 . \mathrm{h}^{-1}$.

\section{Domaine $n^{\circ}(\mathrm{III})$}

Ce domaine correspond une diminution de $(\Phi)$ en fonction de $(M f)$. Cette diminution peut être expliquée par le court-circuitage d'une partie de la surface d'échange externe par la solution à chauffer que l'on observe lors de l'apparition du régime d'excès de débordement (régime hydrodynamique numéro 4). Cette diminution peut également être expliquée par l'augmentation de la 
Tableau 3. Valeurs des grandeurs thermiques obtenues avec l'acide phosphorique à $28 \%$ en $\mathrm{P}_{2} \mathrm{O}_{5}$.

\begin{tabular}{|c|c|c|c|c|c|c|}
\hline \multirow[b]{2}{*}{$\begin{array}{l}M f \\
\left(1 . h^{-1}\right)\end{array}$} & \multirow[b]{2}{*}{$\begin{array}{c}G c \\
\left(\mathrm{~kg} \cdot \mathrm{s}^{-1}\right)\end{array}$} & \multicolumn{2}{|c|}{$\begin{array}{l}\text { Coefficients d'échange } \\
\text { thermique }\left(W \cdot m^{-2} \cdot K^{-1}\right)\end{array}$} & \multicolumn{3}{|c|}{$\begin{array}{c}\text { Résistances thermiques } \\
\left(\mathrm{K} . \mathrm{W}^{-1}\right)\end{array}$} \\
\hline & & $U g$ & $h g f$ & Rji $10^{-3}$ & $\operatorname{Rig} 10^{-3}$ & $\operatorname{Rg} f 10^{-3}$ \\
\hline 290 & $\begin{array}{l}0,607 \\
0,747 \\
0,775 \\
1,012 \\
1,249 \\
\end{array}$ & $\begin{array}{l}682 \\
744 \\
747 \\
875 \\
984 \\
\end{array}$ & 1497 & $\begin{array}{l}1,360 \\
1,437 \\
1,447 \\
1,564 \\
1,662\end{array}$ & 5,726 & 1,374 \\
\hline 459 & $\begin{array}{l}1,097 \\
1,295 \\
1,691 \\
1,935\end{array}$ & $\begin{array}{l}1505 \\
1536 \\
1783 \\
1831\end{array}$ & 2698 & $\begin{array}{l}1,657 \\
1,739 \\
1,867 \\
1,958\end{array}$ & 4,338 & 0,762 \\
\hline 532 & $\begin{array}{l}0,650 \\
0,686 \\
0,814 \\
1,069 \\
1,504 \\
1,658\end{array}$ & $\begin{array}{c}573 \\
883 \\
988 \\
1208 \\
1313 \\
1386\end{array}$ & 2698 & $\begin{array}{l}1,404 \\
1,424 \\
1,498 \\
1,657 \\
1,791 \\
1,842\end{array}$ & 4,338 & 0,762 \\
\hline 747 & $\begin{array}{l}1,390 \\
1,571 \\
1,715 \\
2,287\end{array}$ & $\begin{array}{l}1740 \\
1834 \\
1844 \\
2109\end{array}$ & 3731 & $\begin{array}{l}1,807 \\
1,866 \\
1,913 \\
2,088\end{array}$ & 3,75 & 0,551 \\
\hline
\end{tabular}

résistance au transfert thermique du film en écoulement sur les parois des ailettes (puisque son épaisseur devient de plus en plus importante). Il correspond ici à des valeurs de $M f$ supérieures à $6801 \cdot h^{-1}$.

L'examen de ces résultats, nous permet de faire les commentaires suivants.

L'allure des courbes du coefficient $(U g)$ et de $(\Phi)$ en fonction de $(M f)$, sont semblables (Fig. 11); ceci traduit une certaine proportionnalité entre $U g$ et $\Phi$ si les températures à la sortie ne sont pas imposées, comme c'est le cas de la présente étude.

Les valeurs du coefficient global d'échange $(U g)$ croit avec l'augmentation du débit du fluide de chauffage $(G c)$. En effet l'augmentation de la pression engendre une augmentation de la vitesse de la vapeur de chauffage. Comme l'écoulement de la vapeur est du haut vers le bas le flux de vapeur entraîne avec lui le film de condensât, ce qui affaiblit l'épaisseur de film. Ceci entraîne une diminution de la résistance du film au transfert thermique et par suite l'augmentation du coefficient global d'échange thermique.

Les domaines de variation du coefficient global de transfert thermique $(U g)$ dans nos conditions expérimentales, selon la nature de la solution de chauffage sont :

- l'eau de la ville de Gabès :

$$
630 \mathrm{~W} \cdot \mathrm{m}^{-2} \cdot \mathrm{K}^{-1}<U g<2324 \mathrm{~W} \cdot \mathrm{m}^{-2} \cdot \mathrm{K}^{-1}
$$

- l'acide phosphorique à $28 \%$ en $\mathrm{P}_{2} \mathrm{O}_{5}$ :

$$
245 \mathrm{~W} \cdot \mathrm{m}^{-2} \cdot \mathrm{K}^{-1}<U g<5000 \mathrm{~W} \cdot \mathrm{m}^{-2} \cdot \mathrm{K}^{-1}
$$

- l'acide phosphorique à $54 \%$ en $\mathrm{P}_{2} \mathrm{O}_{5}$ :

$$
300 \mathrm{~W} \cdot \mathrm{m}^{-2} \cdot \mathrm{K}^{-1}<U g<4000 \mathrm{~W} \cdot \mathrm{m}^{-2} \cdot \mathrm{K}^{-1}
$$

Nous pouvons noter que les valeurs du coefficient global d'échange thermique sont deux à trois fois plus grandes que celles d'un échangeur conventionnel à tubes verticaux lisses $[6,13]$.

\subsection{Validation de la méthode indirecte}

En représentant la variation de la résistance globale $(R g)$ en fonction de $(G c)^{-0,8}$ et paramétrée en débit volumique de la solution à chauffer (Fig. 12), nous constatons qu'elle suit des droites qui ne passent pas par l'origine. Nous remarquons aussi que les droites sont quasi parallèles. Ceci confirme la validité de la méthode indirecte.

\subsection{Détermination du coefficient de convection thermique externe (hgf)}

Les résultats du calcul relatif aux résistances et aux coefficients de transfert thermique sont donnés dans le tableau 3.

Le coefficient de transfert par convection paroi externe de l'ailette-film en échauffement ( $h g f$ ) est important. Il est de l'ordre de $2700 \mathrm{~W} . \mathrm{m}^{-2} . \mathrm{K}^{-1}$ pour l'acide phosphorique à $28 \%$ en $\mathrm{P}_{2} \mathrm{O}_{5}$ dans le cas où toutes les ailettes sont mouillées, sans excès de débordement (régime $n^{\circ} 3$ ). 
La figure 13 montre que les résultats de notre calcul indirect par la méthode de WILSON et ceux déterminés expérimentalement par Soetrisnanto [13] sont très semblables pour la gamme des débit d'alimentation entre $4001 \cdot h^{-1}$ et $6001 \cdot h^{-1}$. Cette gamme correspond bien à celle du troisième régime d'écoulement, où toutes les ailettes sont mouillées. Cependant pour les autres régimes la méthode indirecte et celle de la mesure locale directe sont différentes. En effet si l'écoulement sur l'ailette présente des zones sèches ou un excès de débordement, l'écoulement devient complexe et le transfert thermique par convection externe n'est plus homogène sur toute la surface de l'échangeur. Le coefficient de convection paroifilm $(h g f)$ n'est alors plus constant.

Par ailleurs, la valeur de $(h g f)$ ainsi déterminée et injectée dans un calcul numérique comme condition aux limites, permet de prévoir la température ( Tf ) de la solution à chauffer qui s'écoule le long des ailettes. Cette modélisation nous permet de déterminer le numéro de l'ailette où la température est égale à celle de changement de phase, ainsi nous pouvons savoir quelle est la partie de l'échangeur/évaporateur sert à l'échauffement et quelle est la partie sert à l'évaporation. Ce calcul numérique a été validé expérimentalement [2,14].

\section{Conclusion et perspectives}

Cette étude montre l'influence dominante de la nature de l'écoulement sur les performances thermiques de l'échangeur. En particulier, il est intéressant de travailler dans le domaine de la mouillabilité totale des ailettes en évitant toutefois un débordement excessif.

L'étude thermique conduite au cours de ce travail révèle que le tube à ailettes spiralées a un coefficient global d'échange thermique important. En conséquence la hauteur totale et la surface d'échange de cet échangeur/évaporateur seront réduites par rapport à celles d'un évaporateur classique.

Les résultats obtenus pour le calcul du coefficient de convection confirme que la méthode de Wilson est valable et donne des résultats acceptables. Le coefficient de transfert par convection paroi externe de l'ailette-film en échauffement ( $h g f)$ est important. Il est de l'ordre de $2700 \mathrm{~W} \cdot \mathrm{m}^{-2} \cdot \mathrm{K}^{-1}$ pour l'acide phosphorique à $28 \%$ en $\mathrm{P}_{2} \mathrm{O}_{5}$, si toutes les ailettes sont mouillées, sans excès de débordement.

Une fois que le coefficient de transfert par convection est connu nous pouvons faire le bilan thermique convenablement et nous pouvons savoir si les ailettes sont en nombre suffisant pour atteindre l'évaporation.

\section{Références}

[1] Z. Meddeb, M.H. Gazzah, M.R. Jeday, Analyse exergetique d'une unité de concentration de l'acide phosphorique, Phys. Chem. News 12 (2003)

[2] Z. Meddeb, M.R. Jeday, Modélisation du début de l'évaporation dans un nouvel échangeur/évaporateur à ailettes spiralées en graphite, J. Maghrébin de Physique 2(2) (2003) 1-12

[3] P. Le Goff, B. Clauzade, Procédé et dispositif d'échange thermique avec film ruisselant : un nouvel évaporateur à film ruisselant de hautes performances, Brevet d'invention $\mathrm{n}^{\circ}$ 89-15283, France, 1989

[4] Z. Meddeb, M.R. Jeday, Étude expérimentale et théorique de l'écoulement sur des ailettes spiralées, $4^{\mathrm{es}}$ journées sur les Écoulements et les transferts, Hammamet, Décembre 2002

[5] F. Kreith, Transmission de la chaleur et Thermodynamique, Masson, Paris, 1967

[6] P. Wuithier, Raffinage et génie chimique, Édition Technip., Paris, 1965

[7] J.F. Sacadura, Initiation aux transferts thermiques, Édition Technique et documentation ( $4^{\mathrm{e}}$ tirage $)$, Paris, France, 1993

[8] R.E. Wilson, J.R. Welty, C.E. Wicks, Fundamentales of momentum heat and mass transfer, John Wiley \& Sons, New York, 1976

[9] D.E. Briggs, D.L. Katz, E.H. Young, Chemecal. Engineering. Progress., $59 \mathrm{~N}^{\circ} 11,49$ (1963)

[10] D.E. Briggs, E.H. Young, Modified Wilson plot techniques for obtaining heat transfer correlations for shell and tube heat exchangers, Heat transfert - Philadelphia, Chemical Engineering Progress Symposium n ${ }^{\circ}$ 92, vol. 65

[11] R. Bird, W.E. Stewart, E.N. Lightfoot Transport phenomena, John Wiley \& Sons, New York, 1960

[12] J. Gosse, Guide technique de thermique, Bordas, Paris, 1981

[13] A.Y. Soetrisnanto, Un nouvel évaporateur à film ruisselant sur des tubes à ailettes spiralées en graphite, Thèse de Doctorat de l'Institut National Polytechnique de Lorraine à Nancy, France, 1992

[14] Z. Meddeb, M.R. Jeday, The beginning of evaporation in a new Exchanger/Evaporator with helicoidal graphite fins, International Conference on Advances in Mechanical Engineering, Hammamet, Mars, 2002 